\title{
Leveraging early markers of cystic fibrosis structural lung disease to improve outcomes
}

\author{
Patrick A. Flume ${ }^{1}$ and Donald R. VanDevanter ${ }^{2}$ \\ Affiliations: ${ }^{1}$ Depts of Medicine and Pediatrics, Medical University of South Carolina, Charleston, SC, USA. \\ ${ }^{2}$ Dept of Pediatrics, Case Western Reserve University School of Medicine, Cleveland, OH, USA. \\ Correspondence: Patrick A. Flume, Depts of Medicine and Pediatrics, 96 Jonathan Lucas Street, Room 816- \\ CSB, MSC630, Charleston, SC 29451 USA. E-mail: flumepadmusc.edu
}

@ERSpublications

Wijker and colleagues have demonstrated the link of inflammation and obstruction to eventual structural disease in CF. Now we may have another clinical endpoint by which we can judge the impact of early interventions. http://bit.ly/2v5A5Qw

Cite this article as: Flume PA, VanDevanter DR. Leveraging early markers of cystic fibrosis structural lung disease to improve outcomes. Eur Respir J 2020; 55: 2000105 [https://doi.org/10.1183/13993003.001052020].

The natural history of cystic fibrosis (CF) lung disease has been described as a complex process of obstruction, infection, and inflammation, all contributing to airways injury and eventual structural disease (i.e. bronchiectasis). Consequences of the CF genetic abnormality begin in utero and are the basis for newborn screening for elevated levels of circulating immunoreactive trypsinogen (IRT) [1]. Thus, it should be no surprise that the process of airway obstruction begins immediately at birth. Ample cross-sectional evidence has been published of airway obstruction, inflammation, bacterial infection, bronchial wall thickening, air trapping and bronchiectasis in the first year of life for infants with CF [2].

Despite a wealth of observational data, there remain uncertainties as to possible/probable relationships between these different early complications. For instance, there remains some debate as to whether inflammation is inherent to the CF lung, a secondary result of opportunistic microbial infection, or both. Similarly, although there has been an association noted between infection and bronchiectasis in children, reported discordance between the sites of infection and the anatomical site of early bronchiectasis suggest they may not be directly linked [3]. An original study published in this issue of the European Respiratory Journal has now demonstrated that early radiographic lung abnormalities are indeed associated with later structural disease in children with CF [4]. A notable contribution of this work is that of quantitating specific biomarkers of future lung disease risk: atelectasis, airway wall thickening, and increased interleukin (IL)-8 concentrations.

On the one hand, the published results reinforce a CF lung disease progression model that few would find surprising or controversial: that reversible lung obstruction and inflammation in CF preschoolers is highly predictive of subsequent irreversible damage in older children. However, aside from the necessity of proving the hypothesis, it also represents a truly impressive accomplishment resulting from years of dedication to studying early CF lung disease and improving quantitation of chest computed tomography (CT) by Dutch and Australian collaborators. In addition, we now have published guidance for performing CT imaging of CF patients for the purposes of research [5]; by standardising the performance of CT imaging we can reduce observer variability and analytical bias. 
Development and validation of a diagnostic tool for a chronic disease is a necessary first step in a longer process of testing interventions and (hopefully) demonstrating subsequent disease modification. Clarification of temporal associations between early CF lung disease manifestations and subsequent irreversible damage by CT prompts the obvious question of how intervention might be deployed to prevent or delay this process. The mantra has long been that "early intervention" is preferred in order to slow lung disease progression, but there have been doubts as to whether interventions based upon "reversible" CT findings, such as gas trapping, were warranted. Rates of CF lung disease progression are heterogeneous at the population level, making the concept of introducing all respiratory therapies in all patients as early as possible an unappetising proposition. Although aggressive, early-as-possible intervention might benefit some children, it would result in increased burden, cost and risk of adverse events for all children, including those for which little or no benefit would be realised. Our goal must be to improve our precision in therapeutic prescription, initiating therapies in those children for whom benefit is likely. Prescription of inhaled antipseudomonal antibiotics upon the identification of bacteria, notably Pseudomonas, has become standard of care [6], but what of the chronic therapies such as hypertonic saline, dornase alfa and macrolides? The data presented by WIJKER et al. [4] suggest that earlier interventions guided by CT and inflammatory marker findings may be warranted.

Beyond hypothesising that "benefit is likely" in a subpopulation, we should feel obliged to study interventions longitudinally in that population to determine if benefit has occurred, and if so to what extent. This is not a new concept: the challenge has always been how to demonstrate evidence of disease, and by extension, evidence of disease attenuation with treatment, in young children. It is now accepted that children with poor nutritional indices at age 3 years have lower lung function by age 6 years [7] and the trajectory of nutritional status is associated with subsequent pulmonary function [8], justifying nutritional intervention in young children with poor nutritional indices. However, young children lack the tools to tell us how they feel and often lack respiratory symptoms we associate with poor outcomes in older individuals (e.g. cough productive of sputum). In turn, obtaining common functional measures in young children (e.g. forced expiratory volumes) remains realistically possible only in the context of specialised centres. Although abnormal lung clearance index (LCI) measures obtained by multiple breath washout (MBW) in preschool children are predictive of lower lung function later in childhood [9], MBW remains a research tool and has not become a standard procedure in most clinics. Is it time to consider subjecting all our infants with CF to MBW and/or CT imaging to identify those patients showing early signs of progression, or could we be more selective in whom we perform testing? Infants with CF and greater respiratory rates are more likely to have abnormal LCI measures [10], but the difference seems too small to lend itself to diagnostic decisions.

Ideally, we seek demonstration that a therapy can reverse or attenuate abnormal findings of diagnostic tests. Although hypertonic saline inhalation has shown LCI improvements in preschool children [11], it remains to be seen whether consistent hypertonic saline treatments over years will be associated with reduced structural lung disease later in life. This is the next hurdle for CT imaging as an outcome measure: to demonstrate resolution or attenuation of abnormalities following effective interventions. WIJKER et al. [4] have left little doubt that addressing early inflammation in the CF lung will be integral to slowing or stopping CF lung disease progression [4]. Further, their results suggest that studies of intervention-associated change in atelectasis, airway thickening and/or IL-8 concentrations may be justified in infants with CF. Finally, they have provided the methodology for using CT to evaluate the effects of such interventions on lung disease progression in children.

Unfortunately, there are some challenges that the community must face when interpreting and reacting to these results. Perhaps foremost, the early airway inflammation model of CF disease progression seems well-accepted, and it is likely that many if not most subjects in this study were already receiving at least some chronic pulmonary interventions intended to mitigate obstruction and infection. If these risks have been observed in a background of chronic pulmonary interventions, is there a "next level" of intervention available to these children, or are we "doing all we can"?

As we are now in the era of highly effective modulator therapy (HEMT), with drugs that increase the activity of the cystic fibrosis transmembrane conductance regulator (CFTR) activity, this "next level" of intervention has already demonstrated considerable benefits as an "add on" to background therapy. CFTR modulators have been associated with a reduction in the rate of lung function decline [12, 13], suggesting disease modification, presumably by impacting the obstructive aspects of the condition, but perhaps also reducing, or delaying, the infection and inflammatory components. It will be of great interest to observe whether early HEMT intervention will slow the development of structural disease later in life, and it appears that we will now be able to use CT to start these observations at a very early age. 
Sadly, we appear to be on the cusp of a naturalistic study of CFTR modulators in very young children with $\mathrm{CF}$ that may inform this discussion. Although the global CF community likely concurs that there is potential for early modulator access to retard lung disease progression by improving CFTR activity in the airway, the reality is that the cost of these drugs to payers (whether public or private) has produced regional disparities in modulator access to very young children with CF. It may be that a decade from now, it will be possible to use the CT methodologies described by WIJKer et al. [4] to discriminate between those children with CF who enjoyed access to CFTR modulators early in life and those who did not.

Conflict of interest: None declared.

\section{References}

1 Crossley JR, Elliott RB, Smith PA. Dried-blood spot screening for cystic fibrosis in the newborn. Lancet 1979; 1: $472-474$

2 VanDevanter DR, Kahle J, O'Sullivan AK, et al. Cystic fibrosis in young children: a review of disease manifestation, progression, and response to early treatment. J Cyst Fibros 2016; 15: 147-157.

3 Carzino R, Frayman KB, King L, et al. Regional differences in infection and structural lung disease in infants and young children. J Cyst Fibros 2019; in press [https://doi.org/10.1016/j.jcf.2019.10.018].

4 Wijker NE, Vidmar S, Grimwood K, et al. Early markers of cystic fibrosis structural lung disease: follow-up of the ACFBAL cohort. Eur Respir J 2020; 55: 1901694.

5 Van Straten M, Brody AS, Ernst C, et al. Guidance for computed tomography (CT) imaging of the lungs for patients with cystic fibrosis (CF) in research studies. J Cyst Fibros 2019; in press [https://doi.org/10.1016/j.jcf.2019. 09.001].

6 Castellani C, Duff AJA, Bell SC, et al. ECFS best practice guidelines: the 2018 revision. J Cyst Fibr 2018; 17: 153-178.

7 Konstan MW, Butler SM, Wohl MEB, et al. Growth and nutritional indexes in early life predict pulmonary function in cystic fibrosis. J Pediatr 2003; 142: 624-630.

8 Sanders DB, Fink A, Mayer-Hamblett N, et al. Early life growth trajectories in cystic fibrosis are associated with pulmonary function at age 6 years. J Pediatr 2015; 167: 1081-1088.

9 Hardaker KM, Panda H, Hulme K, et al. Abnormal preschool lung clearance index (LCI) reflects clinical status and predicts lower spirometry later in childhood in cystic fibrosis. J Cyst Fibr 2019; 18: 721-727.

10 Korten I, Kieninger E, Yammine S, et al. Respiratory rate in infants with cystic fibrosis throughout the first year of life and association with lung clearance index measured shortly after birth. J Cyst Fibr 2019; 18: 118-126.

11 Ratjen F, Davis SD, Stanojevic S, et al. Inhaled hypertonic saline in preschool children with cystic fibrosis (SHIP): a multicenter, randomized, double-blind, placebo-controlled trial. Lancet Respir Med 2019; 7: 802-809.

12 Sawicki GS, McKone EF, Pasta DJ, et al. Sustained benefit from ivacaftor demonstrated by combining clinical trial and cystic fibrosis patient registry data. Am J Respir Crit Care Med 2015; 192: 836-842.

13 Konstan MW, McKone EF, Moss RB, et al. Assessment of safety and efficacy of long-term treatment with combination lumacaftor and ivacaftor therapy in patients with cystic fibrosis homozygous for the F508del-CFTR mutation (PROGRESS): a phase 3, extension study. Lancet Respir Med 2017; 5: 107-118. 